\title{
Autodesk Meshmixer usado en la enseñanza de la odontología: Es posible?
}

\author{
Autodesk Meshmixer using in dental education: Is it possible? \\ Miguel Saravia-Rojas ${ }^{1, a,}$ Jofre Gutiérrez-Trevejo ${ }^{1, b}$, Mary Fukuhara-Nakama ${ }^{1, c}$, Zulema Velásquez- \\ Huaman 1,d
}

\section{RESUMEN}

La odontologia digital es una realidad, incorporar competencias digitales es ya perentorio en la formación de recurso humano en Odontologia. El uso del software Autodesk Meshmixer es una gran oportunidad para poder introducir tempranamente al estudiante de estomatología. Se comparte la experiencia como en el curso de Laboratorio de la Clínica Integral de Adulto CIA-2021 donde se diseñó una estrategia didáctica para involucrar el manejo de Meshmixer en el modelado 3D o diseño digital en 3D.

PALABRA CLAVE: 3D, Autodesk Meshmixer, educación dental.

\begin{abstract}
Digital dentistry is a reality. Incorporating digital skills is now essential in the training of human resources in Dentistry. Using Autodesk Meshmixer software is a great opportunity to get the stomatology student involved early. The experience is shared as in the CIA I-2021 Adult Comprehensive Clinic Laboratory course a didactic strategy is designed to involve the use of Meshmixer in 3D modeling or 3D digital design.
\end{abstract}

KEYWORD: 3D, Autodesk Meshmixer, dental education

Facultad de Estomatología, Universidad Peruana Cayetano Heredia. Lima, Perú.

Docente Principal, Cirujano Dentista, Maestría en Estomatología, Doctor en Estomatología.

Cirujano Dentista.

Docente Asociado, Cirujano Dentista, Maestría en Estomatología, Maestría en Educación con mención en Docencia e Investigación en Educación Superior.

d Docente Asociado, Cirujano Dentista, Maestría en Estomatología. 


\section{INTRODUCCIÓN}

La aparición del COVID-19 ocasionó que las actividades académicas presenciales fueran suspendidas y se instalen medidas de distanciamiento social. Esto generó que tanto las estrategias de enseñanza, así como los contenidos vertidos en los diferentes cursos sean analizados exhaustivamente.

Al mismo tiempo demandó la incorporación y virtualización de nuevos contenidos.

La implementación de las nuevas tecnologías en la currícula en las facultades de odontología permite revolucionar la forma en la que el conocimiento es percibido y adquirido por los alumnos y que se ven reflejados en los resultados positivos obtenidos en otros estudios. Estas prácticas incentivan a los estudiantes a un aprendizaje autónomo de conceptos teóricos y que, en algunos casos, representa el inicio en las inclinaciones a estudios de posgrado. Además, la inteligencia artificial permite la efectividad del diagnóstico y plan de tratamiento en las diferentes especialidades de la odontología (1-6).

\section{Protocolos Convencionales de la enseñanza de la anatomia dental}

El estudio de la anatomía y morfología de los dientes son fundamentos integrales de una carrera odontológica (7-10).

Además, el conocimiento de las características anatómicas de los dientes también ayuda a comprender su anatomía e interacciones normales, así como a identificar anomalías y patologías del desarrollo (1116).

Los métodos tradicionales de enseñanza de la morfología dental suelen ser:

1. Conferencias o clases magistrales $(7,16)$, respaldadas por prácticas.

2. Sesiones de estudio con dientes extraídos (8-10).

3. Réplica de dientes de plástico (6).

4. Tallado de dientes en cera o tiza (7).

5. Dibujo de imágenes bidimensionales (2D) de dientes $(9,10)$.

Si bien se prefiere estudiar con dientes extraídos, las presiones han aumentado la necesidad de revisar este modelo, incluida la capacidad de obtener y retener un número adecuado de muestras de enseñanza libres de caries o desgaste excesivo, disminución de horas lectivas, integración de los planes de estudio, así como consideraciones éticas y de higiene (5).

En este contexto en el curso de la Clínica Integral del Adulto III-2020 se incorporó el modelado en 3D para zocalados de modelos, troquelado, coronas y puentes dentro del contenido del curso. Esta experiencia permitió a los alumnos adquirir competencias digitales para el diseño y modelado usando el software Autodesk MeshMixer, software para diseño en 3D que cuenta con herramientas muy útiles para facilitar al usuario realizar modelado e impresión de sus diseños. Este aplicativo se encuentra disponible en Windows y Mac OS y es gratuito. Hay que tener presente que Autodesk es una compañía dedicada al software de diseño en 2D y 3D para la industria manufacturera, construcción, entretenimiento y datos transmitidos vía inalámbrica (11).

El aprendizaje asistido por computadora (AAC) se puede utilizar para apoyar la enseñanza tradicional conduciendo a métodos de aprendizaje combinados (11). El uso de AAC proporciona a los usuarios flexibilidad en el aprendizaje, lo que permite controlar el ritmo y revisar (o repetir) el material varias veces al tiempo que reduce la participación del profesorado. Además, un diseño de aprendizaje mixto o combinado no solo proporciona flexibilidad curricular, sino que también aborda las diferencias en los estilos de aprendizaje de los estudiantes (5).

Se ha demostrado que la incorporación de elementos tridimensionales (3D) en la enseñanza de la anatomía favorece la comprensión espacial y la visualización de la relación entre diferentes estructuras (13).

Estudios recientes que exploran el vínculo entre el uso de métodos de enseñanza 3D para mejorar la comprensión de sistemas anatómicos complejos y la capacidad espacial reportado resultados variados con algunos estudios muestran una correlación positiva $(14,15)$, mientras que otros estudios no reportan una correlación significativa (16-19).

La capacidad espacial de los estudiantes de odontología es un predictor del éxito, especialmente en los cursos preclínicos con los estudiantes viendo 
animaciones dinámicas en 3D para mejorar sus modelos mentales. Está claro que los métodos de enseñanza innovadores pueden estimular a los estudiantes y mantener su interés, pero también deben desempeñar un papel didáctico (20).

Meshmixer es una aplicación de modelado en $3 \mathrm{D}$, con la cual se puede crear, reconstruir y esculpir diseños 3D de forma sencilla.

\section{Autodesk Meshmixer}

MeshMixer se basa en mallas triangulares que constan de tres elementos: vértices, aristas y caras o triángulos. Los vértices corresponden a puntos en el espacio 3D, las aristas conectan dos vértices juntos $\mathrm{y}$ las caras corresponden a la asociación de tres vértices (figura 1).

El nombre del software tiene su origen de su primera funcionalidad, Meshmix, es decir, la combinación de mallas $($ mesh $=$ mallas; mixer $=$ mezcla) permite agregar diferentes tipos de mallas usando una herramienta que sirve para arrastrar y soltar. Es uno de los programas más destacados para editar y preparar archivos de tipo malla.

Otra de las herramientas con que cuenta el software es la herramienta "Escultura" que permite al usuario modificar y personalizar sus modelos utilizando diferentes pinceles. Hay dos tipos de herramientas disponibles, volumen (esfera 3D) o superficie (disco 2D).

Otra de las bondades del programa son las funciones de análisis para detectar posibles problemas de impresión. El software facilita el proceso de impresión permitiendo al usuario elegir su impresora 3D. Autodesk Meshmixer es una muy buena plataforma para el modelado y construcción en 3D.

Este software es relativamente fácil de aprender, permite modelar una pieza a partir de otro modelo y está dirigido tanto a estudiantes como a profesionales que se inician en el modelado 3D.

En el mercado de la impresión 3D, el modelado es el primer paso hacia el diseño de una pieza, es la clave que asegura gran parte de su éxito.

Esta experiencia consiguió demostrar que los alumnos de pregrado podían realizar con éxito la práctica y prepararlos de cara a los nuevos retos que el futuro odontólogo debe asumir en relación con la odontología digital que vino para quedarse.

Esta experiencia brindó las bases para que se dé la oportunidad de incorporar el aprendizaje en 3D en la educación dental para el curso Clínica Integral del Adulto I-2021 perteneciente al Departamento Académico de Clínica Estomatológica, curso que

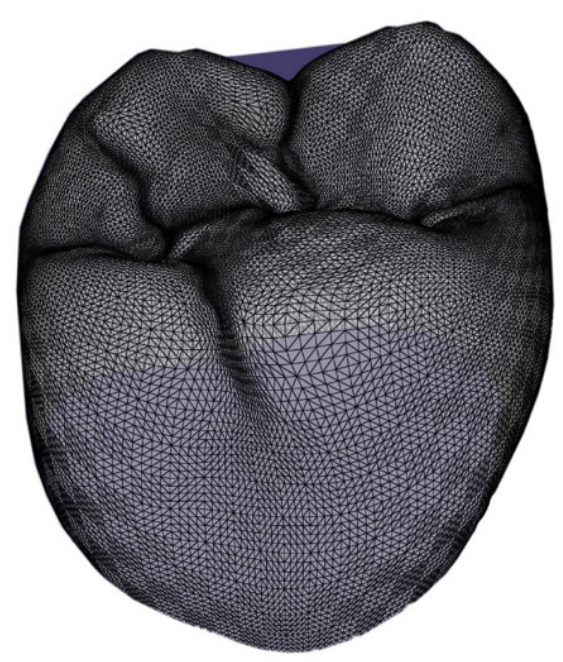

Figura 1. Imagen STL con la disposición de puntos y triángulos 
se dicta en el 5to ciclo de la carrera profesional, en el tópico de encerado o modelado digitalizados de las piezas dentarias, haciendo uso del mencionado software. La principal razón por la que se optó por el uso de este programa es en base a las especificaciones básicas que requiere para su correcto funcionamiento, así como es compatible con los sistemas operativos: Windows y MacOS.

Sin lugar a duda las estrategias didácticas después de la aparición de la COVID-19 han cambiado de manera importante la forma de enseñar y el aprender. Una nueva e innovadora manera de generar aprendizajes para la formación del estudiante de odontología se ha instaurado y con ello la oportunidad de la virtualización de los contenidos, generando nuevas estrategias didácticas y ubicando a nuestra facultad en el liderazgo de la enseñanza de la odontología.

El programa presenta las siguientes herramientas que son de utilidad para el modelado en 3D o encerado digital que a continuación describiremos:

\section{Protocolo de uso:}

Para la actividad de encerado digital o modelado en 3D de las piezas dentarias se elaboró un manual de usos con las distintas herramientas que el programa ofrece:
1. Inflate: Cuyo objetivo es aumentar el volumen de la superficie del sólido a trabajar.

2. Pinch: Que tiene por finalidad la realización de los surcos principales y accesorios.

3. RobustSmooth: Tiene como objetivo alisar la superficie del sólido.

4. ShrinkSmooth: Tiene por finalidad alisar suavemente la textura superficial del sólido.

5. Draw 2: Incrementar la superficie del sólido.

Con esta herramienta se puede generar modelados en 3D o encerados digitales de esculturas tridimensionales trabajando en Autodesk Meshmixer.

Cada alumno contaba con una guía de práctica para poder acompañar las clases sincrónicas, así como para que puedan mejorar el manejo de las herramientas en las distintas tareas que se solicitaban. El protocolo de encerado digital que se elaboró y se utilizó para la elaboración de las esculturas tridimensionales, se denominó "4 Steps / 4 pasos"(figura 2).

Se requería primero la elaboración de un plano base donde los dientes tomarían la forma y característica. El segundo paso consistía en el diseño de los surcos de las piezas dentarias. El tercer paso indica la elaboración de las crestas marginales, cíngulo, cúspides o cualquier estructura que requiera un aumento de volumen. El cuarto paso consistió en

\section{Pasos para encerar:}

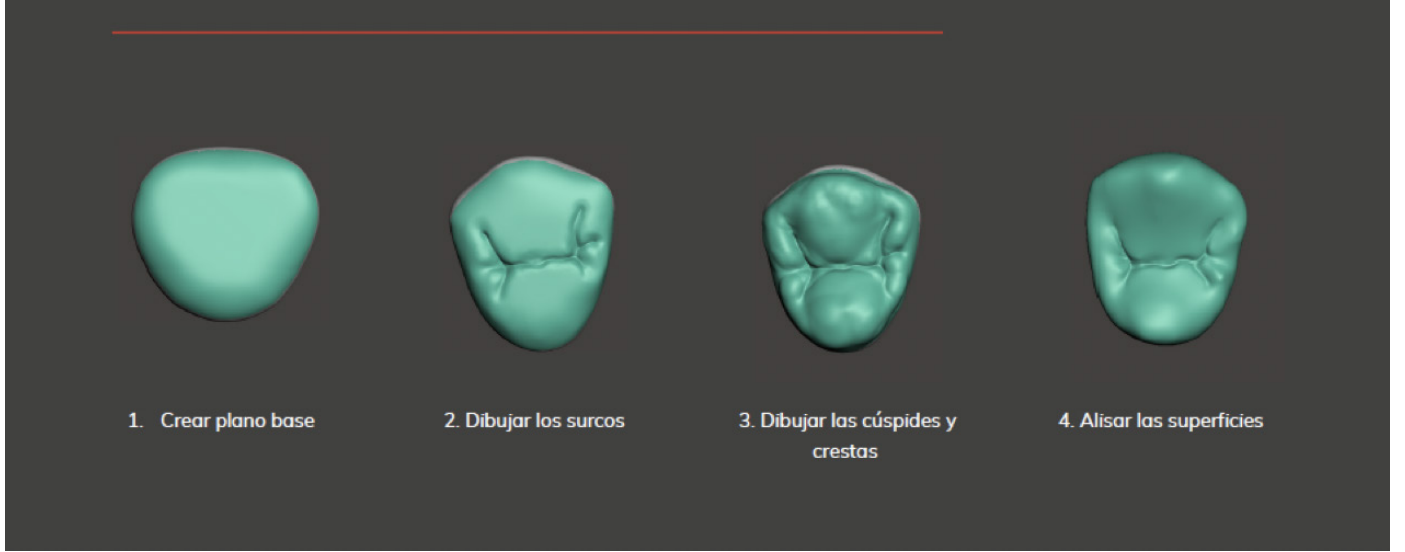

Figura 2. El protocolo de encerado digital "4 Steps / 4 pasos" 
el alisaje de las superficies y perfeccionamiento de las características elaboradas en los pasos anteriores. Para la elaboración de cada pieza dentaria se partió de una esfera (figura predeterminada por el software).

Para la elaboración del plano base se realizaron 2 cortes en la figura inicial (esfera) utilizando las herramientas de la sección "Edit", para el segundo, tercer y cuarto paso solo se utilizaron las herramientas de la sección "Sculpt".

Todos los alumnos recibieron una clase introductoria acerca de los distintos pinceles y configuraciones que se requerían cambiar para brindar características específicas a las piezas dentarias.

Para el segundo paso que consistía en la formación de los surcos principales, se utilizó el pincel "Pinch" debido a que permitía brindar las características similares a las de los surcos de las piezas dentarias. El tercer paso tenía como finalidad generar los aumentos de volumen se utilizó el pincel "Inflate" para brindar toda la característica de las crestas marginales, cúspides, cíngulo, etc. Para el último paso se utilizaron los pinceles "RobustSmooth" y "ShrinkSmooth" para alisar y re-marcar algunas características elaboradas en los pasos anteriores. Las configuraciones de los distintos pinceles fueron brindadas durante las clases sincrónicas.

Las herramientas del Meshmixer permiten esculpir las piezas dentarias en forma similar como se realiza el encerado por aposición tradicional con los instrumentos PKT, a través de incremento de volumen, desgaste, alisado, diseño de surcos y fisuras, entre otros lo que afianzaría sus destrezas para el encerado tradicional, así como para lograr restauraciones más anatómicas.

El uso del software Meshmixer sirvió para diseñar piezas anteriores y posteriores previo a la realización
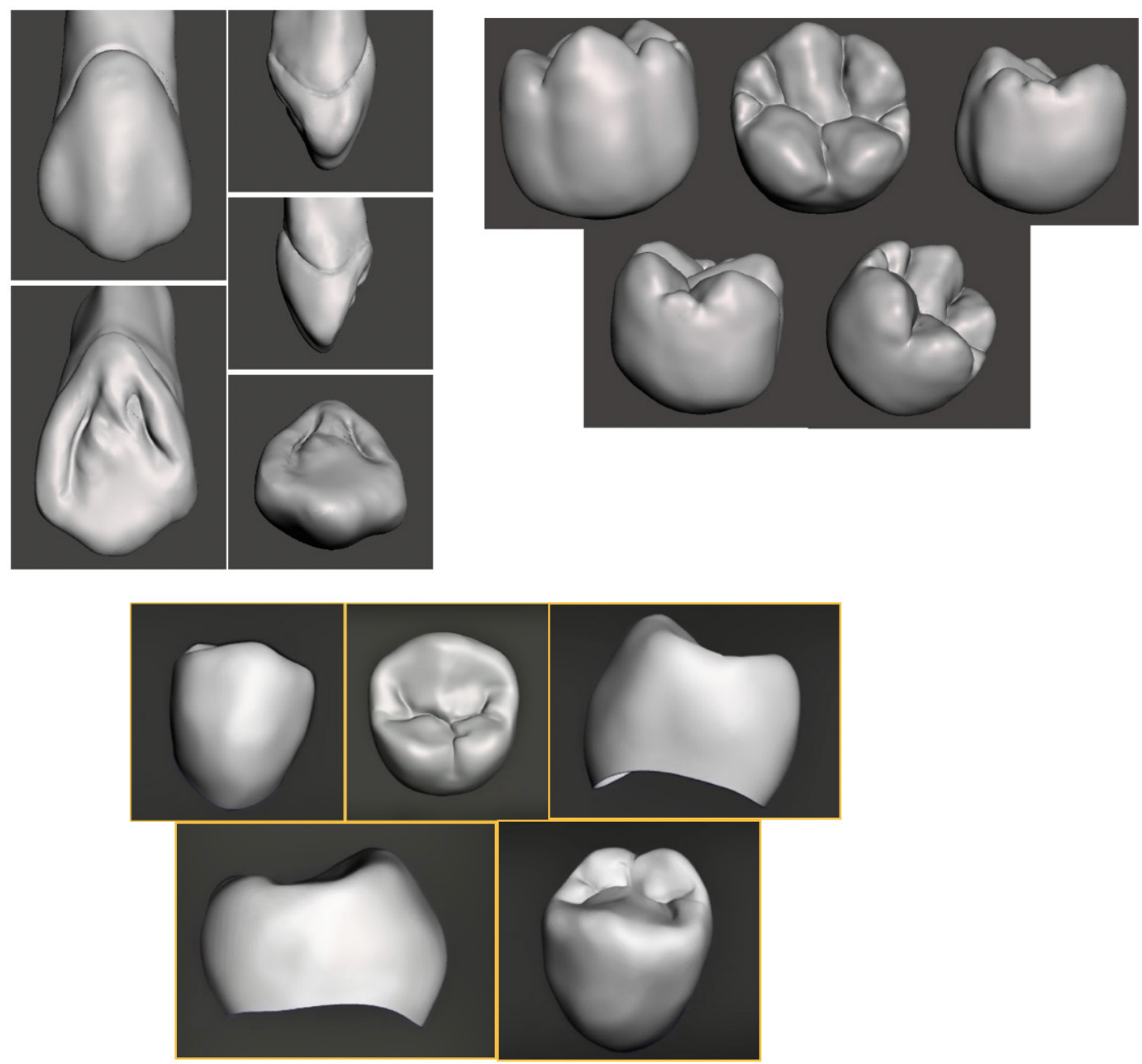

Figura 3. Diseño de piezas dentarias realizadas por estudiantes del tercer año. 
del tradicional encerado por aposición, según estaba indicado el sílabo del curso( figura 3).

\section{Percepción de lo alumnos en el uso de Autodesk Meshmixer}

Con el objetivo de recoger la percepción de los estudiantes sobre esta experiencia en el uso y aplicación de programa de Meshmixer se aplicó una encuesta (validada entre pares) y se encontraron los siguientes resultados:

El $45,9 \%$ de los estudiantes encuestados consideraron que el modelado en 3D utilizando el meshmixer es más fácil de realizar, para el 26.2\% de los estudiantes el encerado por aposición les resultó más fácil y para el $27,9 \%$ el diseño utilizando el meshmixer y el encerado por aposición tenía la misma facilidad en su ejecución.

En relación a la contribución del diseño utilizando el software meshmixer a su aprendizaje de anatomía dentaria, en una escala del 0-5 donde 5 es una máxima contribución: el $37,7 \%$ seleccionó la escala 5, un $45,9 \%$ la escala 4 , es decir el $83,6 \%$ lo considera útil para su aprendizaje.

El 78,6\% considera que realizar primero el diseño en Meshmixer le facilita realizar el encerado por aposición.

En relación con los beneficios de las prácticas el $86,9 \%$ señaló que le ha permitido mejorar su destreza manual, el $80,3 \%$ considera que ha contribuido a ser más detallista, el $65,6 \%$ respondió que le ha permitido introducirse en la odontología digital, al 67,2\% le ha permitido conocer herramientas de diseño en $3 \mathrm{D}$.

Después de esta primera experiencia el 91,8\% considera que debe continuarse con el diseño o modelado en $3 \mathrm{D}$.

\section{CONCLUSIONES}

La introducción del software Autodesk Meshmixer en el aprendizaje del estudiante de odontología es el inicio del camino hacia la necesaria digitalización de la odontología, y la pandemia ha contribuido a mirar más de cerca las herramientas digitales, los estudiantes siendo nativos digitales ven positivamente estas herramientas.
Siendo las funciones de las herramientas del Meshmixer semejantes a las acciones realizadas para ejecutar un encerado por aposición tradicional, la combinación de ambas técnicas contribuiría a mejorar las destrezas del estudiante para lograr encerados y restauraciones más anatómicas. Si es posible su uso en la enseñanza de la odontologia. Será un reto la incorporación de competencias digitales en el cuarto y quinto año de la carrera. La pandemia nos brindó la oportunidad de digitalizar la enseñanza de la odontologia.

\section{Correspondencia}

Zulema Velásquez

Jr. Manuel Villavicencio 420 Urb. Ingeniería

San Martin de Porres, Lima, Perú

Correo electrónico: zulema.velasquez@upch.pe

\section{REFERENCIAS BIBLIOGRÁFICAS}

1. Schlenz MA, Michel K, Wegner K, Schmidt A, Rehmann P, Wöstmann B. Undergraduate dental students' perspective on the implementation of digital dentistry in the preclinical curriculum: a questionnaire survey. BMC Oral Health. 2020;20(1):78

2. Karl M, Franz M, Grobecker-Karl T, Scheib M. Pilot study on the effectiveness and students' attitude towards undergraduate training provided by industry. Eur J Dent Educ. 2018 Aug;22(3):174-178.

3. Khanagar SB, Al-Ehaideb A, Maganur PC, Vishwanathaiah S, Patil S, Baeshen HA, Sarode SC, Bhandi S. Developments, application, and performance of artificial intelligence in dentistry - A systematic review. J Dent Sci. 2021;16(1):508-522.

4. Kato A, Ohno N. Construction of three-dimensional tooth model by micro-computed tomography and application for data sharing. Clin Oral Investig. 2009; 13: 43-46.

5. Cantin M, Muñoz M, Olate S. Generation of 3D tooth models based on three-dimensional scanning to study the morphology of permanent teeth. Int $\mathrm{J}$ Morphol. 2015; 33:782-787.

6. Obrez A, Briggs C, Buckman J, Goldstein L, Lamb C, Knight WG. Teaching clinically relevant dental anatomy in the dental curriculum: Description and assessment of an innovative module. J Dent Educ. 2011; 75:797-804.

7. Bakr MM, Thompson CM, Massadiq M. Anatomical sciences: A foundation for a solid learning experience in dental technology and dental prosthetics. Anat Sci Educ. 2017; 10: 395-404.

8. Mitov G, Dillschneider T, Abed MR, Hohenberg G, Pospiech P. Introducing and evaluating MorphoDent, a Web-based learning program in dental morphology. J Dent Educ. 2010; 74:1133-1139. 
9. Abu Eid R, Ewan K, Foley J, Oweis Y, Jayasinghe J. Self-directed study and carving tooth models for learning tooth morphology: Perceptions of students at the University of Aberdeen, Scotland. J Dent Educ. 2013; 77:1147-1153.

10. Magne P. A new approach to the learning of dental morphology, function, and esthetics: The "2D-3D-4D" concept. Int J Esthet Dent. 2015; 10:32-47.

11. Fernandez E. Autodesk Meshmixer: Herramienta gratuita para modelado en 3D. NEOTEO; 2016. (Citado el 20 de mayo del 2021) Disponible en: https://www. neoteo.com/autodesk-meshmixer-herramientagratuita-para-modelado-en-3d/

12. Maggio MP, Hariton-Gross K, Gluch J. The use of independent, inter- active media for education in dental morphology. J Dent Educ. 2012; 76:1497- 1511.

13. Allen LK, Eagleson R, de Ribaupierre S. Evaluation of an online three-di- mensional interactive resource for undergraduate neuroanatomy education. Anat Sci Educ. 2016; 9:431-439.

14. Estevez ME, Lindgren KA, Bergethon PR. A novel three-dimensional tool for teaching human neuroanatomy. Anat Sci Educ. 2020; 3: 309-317.
15. Hoyek N, Collet C, Di Rienzo F, De Almeida M, Guillot A. Effectiveness of three-dimensional digital animation in teaching human anatomy in an authentic classroom context. Anat Sci Educ. 2014; 7:430-437.

16. Keedy AW, Durack JC, Sandhu P, Chen EM, O’Sullivan PS, Breiman RS. Comparison of traditional methods with 3D computer models in the instruction of hepatobiliary anatomy. Anat Sci Educ. 2011; 4:84-91

17. Vuchkova J, Maybury TS, Farah CS. 211. Testing the educational potential of $3 \mathrm{D}$ visualization software in oral radiographic interpretation. J Dent Educ. 2011; 75:1417-1425.

18. Khot Z, Quinlan K, Norman GR, Wainman B. The relative effectiveness of computer-based and traditional resources for education in anatomy. Anat Sci Educ.2013; 6: 211-215.

19. Preece D, Williams SB, Lam R, Weller R. "Let's get physical": Advantages of a physical model over 3D computer models and textbooks in learning imaging anatomy. Anat Sci Educ. 2013; 6: 216-224.

20. Lone M, McKenna JP, Cryan JF, Downer EJ, Toulouse A. A survey of tooth morphology teaching methods employed in the United Kingdom and Ireland. Eur J Dent Educ. 2018; 22:438-433.

Recibido : 22-06-2021

Aceptado : 28-08-2021 